\title{
Enterocytozoon bieneusi Genotypes and Infections in the Horses in Korea
}

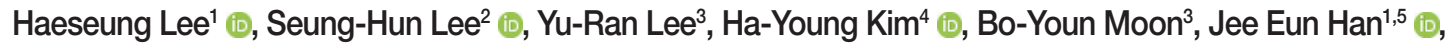 \\ Man Hee Rhee ${ }^{1,5}$ (), Oh-Deog Kwon ${ }^{1}$ (D), Dongmi Kwak ${ }^{1,5, *}$ (]) \\ ${ }^{1}$ College of Veterinary Medicine, Kyungpook National University, Daegu 41566, Korea; ${ }^{2}$ College of Veterinary Medicine, Chungbuk National \\ University, Chungbuk 28644, Korea; ${ }^{3}$ Animal Disease Diagnostic Division, Animal and Plant Quarantine Agency, Gyeongbuk 39660, Korea; ${ }^{4}$ Bacterial \\ Disease Division, Animal and Plant Quarantine Agency, Gyeongbuk 39660, Korea; ${ }^{5}$ Cardiovascular Research Institute, Kyungpook National \\ University, Daegu 41944, Korea
}

\begin{abstract}
Enterocytozoon bieneusi is a microsporidian pathogen. Recently, the equestrian population is increasing in Korea. The horse-related zoonotic pathogens, including E. bieneusi, are concerns of public health. A total of 1,200 horse fecal samples were collected from riding centers and breeding farms in Jeju Island and inland areas. Of the fecal samples 15 (1.3\%) were PCR positive for E. bieneusi. Interestingly, all positive samples came from Jeju Island. Diarrhea and infection in foals were related. Two genotypes (horse1, horse2) were identified as possible zoonotic groups requiring continuous monitoring.
\end{abstract}

Key words: Enterocytozoon bieneusi, genotype, horse, zoonosis

Microsporidiosis caused by Enterocytozoon bieneusi is one of the most important diseases caused by ingesting food or water contaminated with spores and eggs [1]. It can cause life-threatening diarrhea in individuals with weakened immunity and is particularly related to chronic diarrhea in patients with AIDS [2]. E. bieneusi is challenging to observe through a microscope due to its small spore size of about $1 \mu \mathrm{m}$, various shapes, and in vitro culture method $[3,4]$. Therefore, for detecting and genotyping E. bieneusi, sequencing analysis by PCR of the ribosomal internal transcribed spacer (ITS) locus of E. bieneusi was used $[3,5]$.

Enterocytozoon bieneusi was identified from livestock, companion animals, primates, wildlife, birds, and even wastewater $[1,3]$. By sequencing analysis of the ITS region of E. bieneusi, more than 500 genotypes have been identified and divided into 11 genetic groups $[5,6]$. Group 1 is the largest, while group 2 is the second largest group. These 2 groups are the most zoonotic-potential, and groups 3-11 mainly exhibit host specificity but lacks information [3,5]. More than 40 geno-

- Received 22 November 2021, revised 28 November 2021. accepted 28 November 2021

*Corresponding author (dmkwak@knu.ac.kr) (c) 2021, Korean Society for Parasitology and Tropical Medicine This is an Open Access article distributed under the terms of the Creative Commons Attribution Non-Commercial License (https://creativecommons.org/licenses/by-nc/4.0) which permits unrestricted non-commercial use, distribution, and reproduction in any medium, provided the original work is properly cited. types of $E$. bieneusi in equine have been identified, and classified into groups $1,2,6$, and 10 [3].

Prevalence of $E$. bieneusi in equine was previously studied in several countries [7-11]. In Korea, several studies on E. bieneusi in other animals were performed [12-15] but no report from horses. The horse industry in Korea is expanding to horse breeding and leisure areas such as horse racing and horseback riding. Accordingly, the number of horses and people who encounter horses is increasing. Therefore, research on horse-related zoonotic pathogens, including E. bieneusi, is an important issue. This study surveyed the infection of E. bieneusi in horses and assessed its genotype.

From January 2019 to May 2021, 1,200 horse fecal samples were collected each from individual horses at riding centers and breeding farms, mainly in Jeju Island and other inland areas. This procedure was performed by experienced veterinarians, caused no harm to the animals, and did not require ethical approval. For data analysis, we requested to record basic information, such as age, sex, region, and collection date. The fecal samples with little or unclear information were classified as "unknown."

Genomic DNA was extracted using a commercial kit from Qiagen (Hilden, Germany), all processes were performed according to the manufacturer's protocol. The quality and quantity were measured using the Infinite 200 PRO NanoQuant 
plate reader (Tecan, Mannedorf, Switzerland). E. bieneusi was detected by nested PCR amplification of the ITS region using the AccuPower HotStart PCR Premix (Bioneer, Daejon, Korea). PCR was performed using primers and thermal cycling conditions used in previous studies $[2,14]$. The expected amplicon was about $390 \mathrm{bp}$ [16]. All PCR products were electrophoresed in 1.5\% agarose gel and stained with ethidium bromide.

For DNA sequencing and phylogenetic analysis, all PCRpositive samples were sequenced bidirectionally by Macrogen Co. (Daejeon, Korea). The nucleotide sequences were aligned, and consensus sequences were acquired using BioEdit 7.2.5 [17] and MEGA 7 [18]. Phylogeny was performed using MEGA 7 with the maximum-likelihood method. A phylogenetic tree was assessed by 1,000 replicate bootstrap analyses.

An overall E. bieneusi positive rate was 1.3\% $(15 / 1,200)$ (Table 1). It was low compared with the infection rate in other animals previously studied in Korea. The infection was reported in animals such as Korean water deer 53.6\%, raccoon dogs $35.4 \%$, calves $16.9 \%$, cattle $14.9 \%$, piglets $14.2 \%$, bats $5.2 \%$, cats 3.8\%, and wild boar 2.6\% [12-15,19-21]. In horses, E. bieneusi infection rate was lower in Korea than in other countries

Table 1. Enterocytozoon bieneusi infection rates according to different variables

\begin{tabular}{|c|c|c|c|c|c|}
\hline \multirow{2}{*}{ Variable } & \multirow{2}{*}{$\begin{array}{l}\text { No. } \\
\text { tested }\end{array}$} & \multirow{2}{*}{$\begin{array}{c}\text { Positive } \\
\text { (\%) }\end{array}$} & \multirow{2}{*}{$P$-value* } & \multicolumn{2}{|c|}{ Genotypic group (n) } \\
\hline & & & & Group 1 & Group 6 \\
\hline \multicolumn{6}{|l|}{ Age (yr) } \\
\hline$\leq 1$ & 123 & $9(7.3)$ & $<0.001$ & 4 & 5 \\
\hline $2-5$ & 343 & 0 & & - & - \\
\hline $6-10$ & 314 & 0 & & - & - \\
\hline$\geq 11$ & 223 & $1(0.5)$ & & 1 & - \\
\hline Unknown & 197 & $5(2.5)$ & & 2 & 3 \\
\hline \multicolumn{6}{|l|}{ Sex } \\
\hline Female & 804 & $7(0.9)$ & 0.077 & 4 & 3 \\
\hline Male & 222 & $3(1.4)$ & & 1 & 2 \\
\hline Unknown & 174 & $5(2.9)$ & & 2 & 3 \\
\hline \multicolumn{6}{|l|}{ Fecal type } \\
\hline Diarrhea & 92 & $5(5.4)$ & 0.004 & 2 & 3 \\
\hline Normal & 1,108 & $10(0.9)$ & & 5 & 5 \\
\hline \multicolumn{6}{|l|}{ Season } \\
\hline Spring & 395 & $10(2.5)$ & 0.032 & 5 & 5 \\
\hline Summer & 483 & $5(1.0)$ & & 2 & 3 \\
\hline Autumn & 111 & 0 & & - & - \\
\hline Winter & 211 & 0 & & - & - \\
\hline \multicolumn{6}{|l|}{ Region } \\
\hline Jeju & 923 & 15 (1.6) & 0.029 & 7 & 8 \\
\hline Inland & 277 & 0 & & - & - \\
\hline Total & 1,200 & $15(1.3)$ & & 7 & 8 \\
\hline
\end{tabular}

*Significant $(P<0.05)$ correlation with infection.
(Colombia 10.8\%, China 30.9\%, Czech Republic 17.3\%, Turkey $18.7 \%)[8-10,22]$.

In this study, chi-square test was performed using the statistical package for social sciences (v.26, SPSS Inc., Chicago, Illinois, USA), and statistically significant results were obtained for all variables except sex $(P<0.05)$. Most positive samples for age were from the $\leq 1$ year group, 1 sample was from $\geq 11$ years group, and 5 samples were of unknown age $(P<0.001)$. Infection rate of these groups were $7.3 \%$ (9/123), 0.5\% (1/223), and 2.5\% (5/197), respectively. Similar cases were reported in Colombian equid species ( $\leq 1$ year, 23.7\%; $>1$ year, $2.5 \%$ ) and in Chinese horses ( $\leq 1$ year, $32.6 \%$; $>1$ year, $30.6 \%)[8,9]$. It was reported that the older group has a higher infection rate than the younger group in Czech Republic $(\leq 3$ years, $15.7 \%$; $>3$ years, 19.6\%) and in Turkey ( $\geq 4$ years, $19.7 \%$; $1-3$ years, $16.3 \%)[10,22]$. There was no difference in sex (female, $0.9 \%$; male, 1.4\%), which agrees with previous studies [8-10]. Association with diarrhea was higher in diarrheal than in normal ones (5.4\% vs. $0.9 \%)$. E. bieneusi infection was confirmed in the spring $(10 / 395 ; 2.5 \%)$ and summer $(5 / 483 ; 1.0 \%)$. However, this does not mean that there was no infection in autumn and winter, but previous studies showed infection in the spring and summer [23-25]. According to regional data, all positive samples were confirmed in Jeju Island $(15 / 923 ; 1.6 \%)$. Jeju is the largest island on the Korean peninsula and located in the southernmost part. Its climate is warmer than in mainland Korea. However, number of positive fecal samples was small, and more than half of the Korean horses were bred in Jeju [26]. Therefore, whether E. bieneusi infection of horses is specific to the Jeju region needs further study.

By sequencing, 3 representative sequences were obtained from 15 PCR-positive products and submitted to GenBank under accession numbers MW753227-MW753229. A phylogenetic tree confirmed 2 genotypes, horse1 and horse2, belonging to groups 1 and 6 (Fig. 1). Group 6 comprised of 8 samples, while group 1 of 7 samples. There was no mixed infection of the E. bieneusi genotypes.

Group 1 is the largest group divided into 9 subgroups (1a1i), with more than 300 genotypes identified [3], in various animals, including horses and humans. Group 1 is common in horses. Horse E. bieneusi genotypes were 1a, 1b/c, 1d, 1e, and $1 \mathrm{~g}$ subgroups [3]. In horses, E. bieneusi was also identified in group 6, and genotypes clustered into groups 2 and 10 $[8,10,27]$. This study identified the E. bieneusi genotypes clustered into subgroup 1e (MW753227) and group 6 (MW753228, 
MW753229) (Fig. 1). Subgroup 1e was identified several times in other animals in Korea $[12,19,21]$, but the group 6 genotype was identified for the first time in this study. The sequence identified as subgroup 1e was 100\% identical to the sequence reported in Colombian horse (GQ406053) and the Chinese Monkey (KX905206), and 98.9\% similar to E. bieneusi in Indi- ans (KC708073). This showed $97.4 \%$ sequence similarity with genotype 1e in Korean wild boar (MT984295), suggesting low level of host specificity and zoonotic potential [3].

Group 6 genotype has been found in animals (equine, ruminants, nonhuman primates, rodents) and humans [7,2831]. This genotype has strong host specificity since it has been

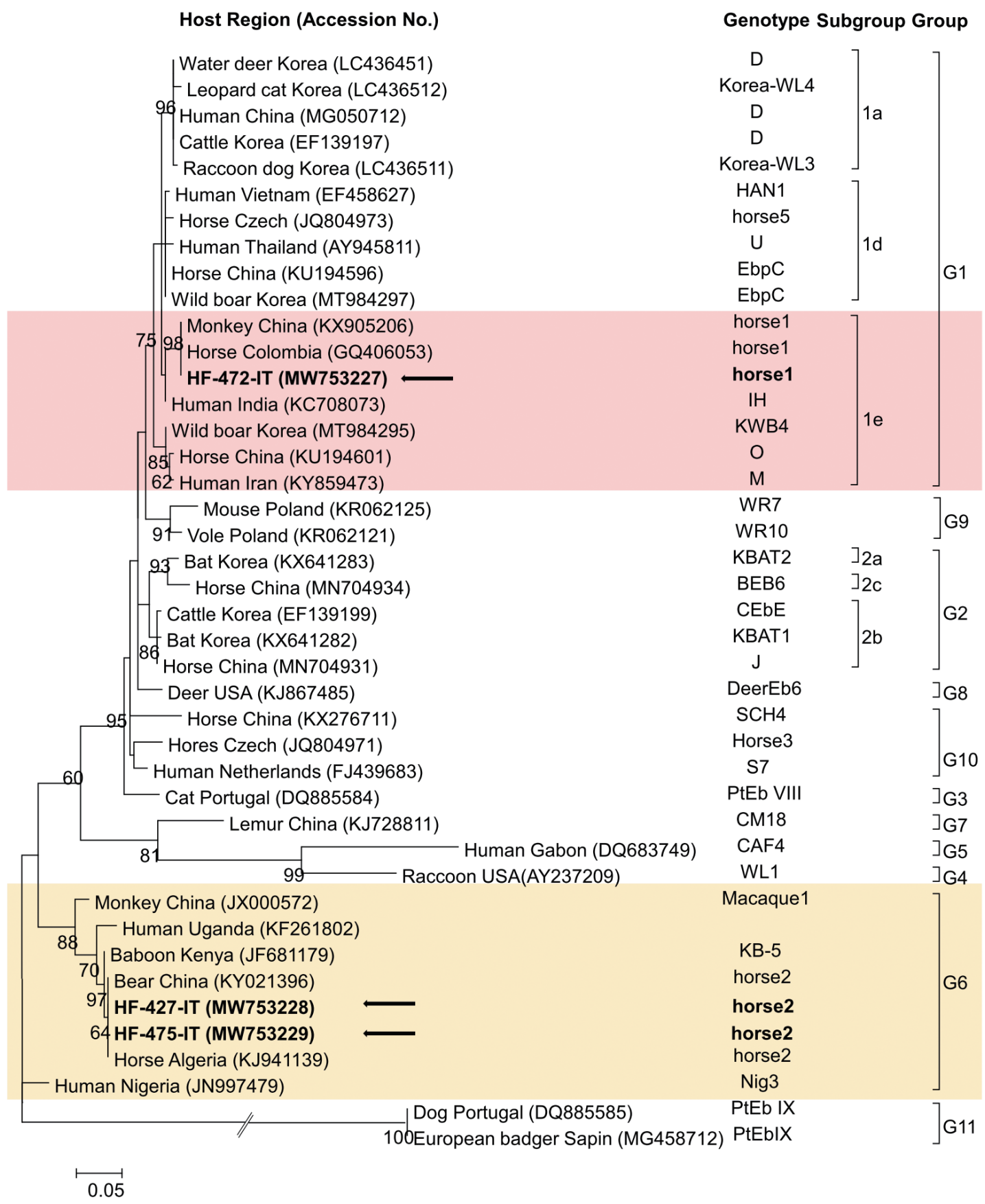

Fig. 1. A phylogenic tree figured based on the internal transcribed spacer sequence of Enterocytozoon bieneusi detected from the horses. Sequences identified in this study are indicated using arrows. Genotypes (groups and subgroups) are shown on right. 
identified only in the originally reported host species, but new hosts for the same genotype have recently been identified $[3,30,32]$. Therefore, the group 6 subgenotypes were suggested with zoonotic potential. The group 6 sequence identified in this study (MW753228) showed 100\% identity with the sequence identified in Chinese bear (KY021396) and 99.7\% identity to sequence in the Algerian horse (KJ941139). The horses may play a potential role as a reservoir for zoonotic transmission since they showed $97.4 \%$ sequence similarity to E. bieneusi in the Ugandans (KF261802).

This is the first study of $E$. bieneusi infection in horses in Korea, providing basic public health data related to E. bieneusi infection pattern and rate. The results indicated that young age and diarrhea were associated with infection. Although the infection rate was low, a positive test result was observed only in the Jeju Island. This research is needed to be continued since both groups had zoonotic transmission potential.

\section{ACKNOWLEDGMENTS}

This work was supported by grants from the Animal and plant quarantine agency (B-1543069-2019-21-03), Korea, and from the Basic Science Research Program through the National Research Foundation of Korea funded by the Ministry of Education (NRF-2016R1D1A1B02015366).

\section{CONFLICT OF INTEREST}

The authors declare no conflict of interest related to this study.

\section{REFERENCES}

1. Feng S, Jia T, Huang J, Fan Y, Chang H, Han S, Luo J, He H. Identification of Enterocytozoon bieneusi and Cryptosporidium spp. in farmed wild boars (Sus scrofa) in Beijing, China. Infect Genet Evol 2020; 80: 104231. https://doi.org/10.1016/j.meegid.2020.104231

2. Karim MR, Dong H, Li T, Yu F, Li D, Zhang L, Li J, Wang R, Li S, Li X, Rume FI, Ning C. Predomination and new genotypes of Enterocytozoon bieneusi in captive nonhuman primates in zoos in China: high genetic diversity and zoonotic significance. PLoS One 2015; 10: e0117991. https://doi.org/10.1371/journal.pone.0117991

3. Li W, Feng Y, Santin M. Host specificity of Enterocytozoon bieneusi and public health implications. Trends Parasitol 2019; 35: 436451. https://doi.org/10.1016/j.pt.2019.04.004

4. Li W, Feng Y, Zhang L, Xiao L. Potential impacts of host specificity on zoonotic or interspecies transmission of Enterocytozoon bieneusi.
Infect Genet Evol 2019; 75: 104033. https://doi.org/10.1016/j. meegid.2019.104033

5. Ou Y, Jiang W, Roellig DM, Wan Z, Li N, Guo Y, Feng Y, Xiao L. Characterizations of Enterocytozoon bieneusi at new genetic loci reveal a lack of strict host specificity among common genotypes and the existence of a canine-adapted Enterocytozoon species. Int J Parasitol 2021; 51: 215-223. https://doi.org/10.1016/j.ijpara. 2020.09 .008

6. Dong H, Cheng R, Li X, Li J, Chen Y, Ban C, Zhang X, Liu F, Zhang L. Molecular identification of Cryptosporidium spp., Enterocytozoon bieneusi, and Giardia duodenalis in captive pet birds in Henan province, central China. J Eukaryot Microbiol 2021; 68: e12839. https://doi.org/10.1111/jeu.12839

7. Laatamna AE, Wagnerová $\mathrm{P}$, Sak B, Květoňová D, Xiao L, Rost M, McEvoy J, Saadi AR, Aissi M, Kváč M. Microsporidia and Cryptosporidium in horses and donkeys in Algeria: detection of a novel Cryptosporidium hominis subtype family (Ik) in a horse. Vet Parasitol 2015; 208: 135-142. https://doi.org/10.1016/j.vetpar.2015.01.007

8. Qi M, Wang RJ, Wang HY, Jian FC, Li JQ, Zhao JF, Dong HJ, Zhu HL, Ning CS, Zhang LX. Enterocytozoon bieneusi genotypes in grazing horses in China and their zoonotic transmission potential. J Eukaryot Microbiol 2016; 63: 591-597. https://doi.org/10.1111/ jeu. 12308

9. Santin M, Vecino JA, Fayer R. A zoonotic genotype of Enterocytozoon bieneusi in horses. J Parasitol 2010; 96: 157-161. https://doi. org/10.1645/GE-2184.1

10. Wagnerová $P$, Sak B, Květoňová $D$, Buňatová $Z$, Civišová $H$, Maršálek M, Kváč M. Enterocytozoon bieneusi and Encephalitozoon cuniculi in horses kept under different management systems in the Czech Republic. Vet Parasitol 2012; 190: 573-577. https:// doi.org/10.1016/j.vetpar.2012.07.013

11. Wagnerová P, Sak B, McEvoy J, Rost M, Sherwood D, Holcomb K, Kváč M. Cryptosporidium parrum and Enterocytozoon bieneusi in American mustangs and Chincoteague ponies. Exp Parasitol 2016; 162: 24-27. https://doi.org/10.1016/j.exppara.2015.12.004

12. Amer S, Kim S, Han JI, Na KJ. Prevalence and genotypes of Enterocytozoon bieneusi in wildlife in Korea: a public health concern. Parasit Vectors 2019; 12: 160. https://doi.org/10.1186/s13071-0193427-6

13. Hwang S, Shin SU, Kim S, Ryu JH, Choi KS. Zoonotic potential of Enterocytozoon bieneusi in pre-weaned Korean native calves. Parasit Vectors 2020; 13: 300. https://doi.org/10.1186/s13071-020-041752

14. Lee H, Seo MG, Lee SH, Oem JK, Kim SH, Jeong H, Kim Y, Jheong WH, Kwon OD, Kwak D. Distribution and genotypic analysis of Enterocytozoon bieneusi from wild boars in Korea. Med Mycol 2021; 59: 934-938. https://doi.org/10.1093/mmy/myab030

15. Lee SH, Oem JK, Lee SM, Son K, Jo SD, Kwak D. Molecular detection of Enterocytozoon bieneusi from bats in South Korea. Med Mycol 2018; 56: 1033-1037. https://doi.org/10.1093/mmy/myx136

16. Zhang XX, Cong W, Lou ZL, Ma JG, Zheng WB, Yao QX, Zhao Q, Zhu XQ. Prevalence, risk factors and multilocus genotyping of Enterocytozoon bieneusi in farmed foxes (Vulpes lagopus), Northern 
China. Parasit Vectors 2016; 9: 72. https://doi.org/10.1186/s13071016-1356-1

17. Hall TA. BioEdit: a user-friendly biological sequence alignment editor and analysis program for Windows 95/98/NT. Nucleic Acids Symp Ser 1999; 41: 95-98.

18. Kumar S, Stecher G, Tamura K. MEGA7: molecular evolutionary genetics analysis version 7.0 for bigger datasets. Mol Biol Evol 2016; 33: 1870-1874. https://doi.org/10.1093/molbev/msw054

19. Jeong DK, Won GY, Park BK, Hur J, You JY, Kang SJ, Oh IG, Lee YS, Stein BD, Lee JH. Occurrence and genotypic characteristics of Enterocytozoon bieneusi in pigs with diarrhea. Parasitol Res 2007; 102: 123-128. https://doi.org/10.1007/s00436-007-0740-3

20. Kwak D, Seo MG. Genetic analysis of zoonotic gastrointestinal protozoa and microsporidia in shelter cats in South Korea. Pathogens 2020; 9: 894. https://doi.org/10.3390/pathogens9110894

21. Lee JH. Prevalence and molecular characteristics of Enterocytozoon bieneusi in cattle in Korea. Parasitol Res 2007; 101: 391-396. https://doi.org/10.1007/s00436-007-0468-0

22. Yildirim A, Okur M, Uslug S, Onder Z, Yetismis G, Duzlu O, Ciloglu A, Simsek E, Inci A. First report on the molecular prevalence of Enterocytozoon bieneusi in horses in Turkey: genotype distributions and zoonotic potential. Parasitol Res 2020; 119: 28212828. https://doi.org/10.1007/s00436-020-06783-4

23. Feng Y, Gong X, Zhu K, Li N, Yu Z, Guo Y, Weng Y, Kváč M, Feng Y, Xiao L. Prevalence and genotypic identification of Cryptosporidium spp., Giardia duodenalis and Enterocytozoon bieneusi in preweaned dairy calves in Guangdong, China. Parasit Vectors 2019; 12: 41. https://doi.org/10.1186/s13071-019-3310-5

24. Zhang Y, Koehler AV, Wang T, Haydon SR, Gasser RB. Enterocytozoon bieneusi genotypes in cattle on farms located within a water catchment area. J Eukaryot Microbiol 2019; 66: 553-559. https:// doi.org/10.1111/jeu.12696

25. Zhang Y, Mi R, Yang J, Wang J, Gong H, Huang Y, Wang X, Han X, Zhou H, Chen Z. Enterocytozoon bieneusi genotypes in farmed goats and sheep in Ningxia, China. Infect Genet Evol 2020; 85: 104559. https://doi.org/10.1016/j.meegid.2020.104559

26. Ministry of Agriculture, Food and Rural Affairs, South Korea (MAFRA). Report of "A Fact Finding Survey of Horse Industry in South Korea during 2019”. 2020.

27. Deng L, Li W, Zhong ZJ, Gong C, Liu XH, Huang XM, Xiao L, Zhao RX, Wang WY, Feng F, Zhang Y, Hu YC, Fu HL, He M, Zhang Y, Wu KJ, Peng GN. Molecular characterization and multilocus genotypes of Enterocytozoon bieneusi among horses in southwestern China. Parasite Vector 2016; 9: 561. https://doi.org/10.1186/ s13071-016-1844-3

28. Akinbo FO, Okaka CE, Omoregie R, Dearen T, Leon ET, Xiao L. Molecular epidemiologic characterization of Enterocytozoon bieneu$s i$ in HIV-infected persons in Benin City, Nigeria. Am J Trop Med Hyg 2012; 86: 441-445. https://doi.org/10.4269/ajtmh.2012.11-0548

29. Baroudi D, Zhang H, Amer S, Khelef D, Roellig DM, Wang Y, Feng Y, Xiao L. Divergent Cryptosporidium parrum subtype and Enterocytozoon bieneusi genotypes in dromedary camels in Algeria. Parasitol Res 2018; 117: 905-910. https://doi.org/10.1007/ s00436-017-5734-1

30. Deng L, Li W, Yu X, Gong C, Liu X, Zhong Z, Xie N, Lei S, Yu J, $\mathrm{Fu} \mathrm{H}$, Chen H, Xu H, Hu Y, Peng G. First report of the humanpathogenic Enterocytozoon bieneusi from red-bellied tree squirrels (Callosciurus erythraeus) in Sichuan, China. PLoS One 2016; 11: e0163605. https://doi.org/10.1371/journal.pone.0163605

31. Ye J, Xiao L, Ma J, Guo M, Liu L, Feng Y. Anthroponotic enteric parasites in monkeys in public park, China. Emerg Infect Dis 2012; 18: 1640-1643. https://doi.org/10.3201/eid1810.120653

32. Deng L, Li W, Zhong Z, Gong C, Cao X, Song Y, Wang W, Huang X, Liu X, Hu Y, Fu H, He M, Wang Y, Zhang Y, Wu K, Peng G. Multilocus genotypes of Enterocytozoon bieneusi in captive Asiatic black bears in southwestern China: high genetic diversity, broad host range, and zoonotic potential. PLoS One 2017; 12: e0171772. https://doi.org/10.1371/journal.pone.0171772 
